\title{
Beat-to-beat estimate of fetal cardiac time intervals using magnetocardiography: longitudinal charts of normality ranges and individual trends
}

\author{
Silvia Comani ${ }^{1,2}$, Marco Liberati ${ }^{3}$, Dante MantinI ${ }^{4}$, Biagio Merlino ${ }^{5}$, Giovanna Alleva ${ }^{1}$, Elisabetta Gabriele ${ }^{3}$, \\ Silvano Di Luzio ${ }^{1,2}$ and Gian Luca Romani ${ }^{1,2}$ \\ From the 'ITAB-Institute of Advanced Biomedical Technologies, University Foundation 'G. D'Annunzio', \\ ${ }^{2}$ Department of Clinical Sciences and Bio-imaging, ${ }^{3}$ Department of Medical Sciences, Chieti University, \\ ${ }^{4}$ Department of Informatics and Automation Engineering, Marche Polytechnic University, Ancona, and \\ ${ }^{5}$ Institute of Radiology, Catholic University, Rome, Italy
}

\section{Acta Obstet Gynecol Scand 2005; 84: 1175-1180. (C) Acta Obstet Gynecol Scand 842005}

Background. Fetal magnetocardiography (fMCG) records fetal cardiac electro-physiological activity during the second half of gestation. We aimed at assessing normality values, related variability, and trends of fetal cardiac time intervals (fCTI) evaluated longitudinally from beat-to-beat fMCG analysis in uncomplicated pregnancies.

Materials and methods. The fMCG were recorded with multi-channel system in shielded room. FCTI were estimated on more than 2600 fetal cardiac cycles from $51 \mathrm{fMCG}$ data sets of uncomplicated pregnancies. Independent component analysis (ICA) allowed reconstructing reliable fetal signals for beat-to-beat identification of fCTI (RR, P wave, PQ, PR, QT, QTc, QRS, ST, and T wave); intra-individual variability analysis and trends were calculated; reference longitudinal charts accounted for intra- and interindividual variations and were compared with figures estimated on averaged signals.

Results. For each data set, fCTI were calculated beat-to-beat on rhythm strips of more than 50 beats $(95 \%$ overall detection rate). FCTI values, variability, and trends were in good agreement with available reference figures; intervals related to $\mathrm{P}$ and $\mathrm{T}$ waves were, respectively, underestimated and overestimated with respect to those estimated on averaged signals or obtained by other research groups. Errors were reduced and individual trends could be drawn.

Conclusions. ICA permitted the reconstruction of reliable time course of fetal cardiac signals and the beat-to-beat calculation of time intervals, and normality ranges, with smaller errors with respect to previous studies. The retrieval of fetal traces with clear morphology and the longitudinal character of the study allowed estimating individual trends and beat-tobeat characterization, impossible with cross-sectional studies on averaged beats.

Key words: Fetal cardiac time intervals (fCTI); fetal magnetocardiography (fMCG); independent component analysis (ICA); signal processing; prenatal diagnosis

Submitted 26 July, 2004

Accepted 26 February, 2005

\section{Abbreviations:}

dc-SQUID: dc-Super-conducting quantum interference device; FastICA: Fixed-Point ICA algorithm; fECG: fetal electrocardiogram; FMCG: fetal magnetocardiography; fT: femto Tesla; Hz: Hertz; kHz: kilo Hertz; ICA: independent component analysis; ms: milliseconds; $\mathrm{ms}^{1 / 2}$ : square root of milliseconds; pT: pico Tesla; SD: standard deviation; SSD: sampled standard deviation.
Fetal magnetocardiography (fMCG) allows the non-invasive registration of fetal heart electrical activity by measuring the associated magnetic field variations above the maternal abdomen (1). No other technique, presently available in the clinical practice, allows monitoring the fetal cardiac electrophysiology in a reliable way, in 
particular during the second half of gestation $(2,3)$. FMCG is preferable to trans-abdominal fetal electrocardiogram (fECG), because it is almost unaffected by the insulating properties of vernix caseosa (4), and it was proved to provide, after adequate postprocessing, a trace coincident with fECG measured with scalp electrodes during labor, allowing precise identification of the onset and offset of P, QRS, and $\mathrm{T}$ waves and therefore enabling the quantification of fetal cardiac time intervals (fCTI) (5). For this reason, fMCG has been used in several clinical situations, such as fetal arrhythmias (6-9), congenital heart defects (10), growth-restricted fetuses (11), cardiac hypertrophy in fetuses of diabetic mothers (12), and fetal hypoxia (13).

Grimm et al. (11) observed that growth restricted fetuses show fCTI different from those of normally grown fetuses: therefore, it might be interesting to evaluate the prognostic value of fCTI changes during pregnancy and, in order to achieve an early diagnosis, to establish whether any abnormalities were present before fetal growth restriction became evident.

From this perspective, the definition of precise and reliable normal variability ranges for all fMCG parameters is of outmost importance. Several authors reported normality data of fCTI at different gestational ages obtained from crosssectional studies on averaged fetal beats of a large number of patients evaluated once in their pregnancy (14-16). However, it is our opinion that, given the high variability of fetal electrical and associated magnetic events, it would be desirable to monitor the fetal cardiac function on longitudinally sequences of well-defined cardiac cycles during the same pregnancies in order to achieve more comprehensive sets of reference values.

In the present study, we longitudinally followed a group of normal fetuses of uncomplicated pregnancies; for each patient fMCG signals were recorded every 4-6 weeks, and clear and reliable fetal traces were reconstructed from each data set by means of independent component analysis (ICA), which has already demonstrated its effectiveness to this purpose $(17,18)$. Therefore, a beat-to-beat analysis of fetal cardiac signals could be performed for each acquisition session, and fCTI variability was evaluated at an individual level during the second half of pregnancy. Furthermore, longitudinal charts were obtained by grouping the outcome of the analysis on single patients in function of gestational age.

\section{Materials and methods}

The protocol, approved by the Ethical Committee of our University, consisted of a longitudinal evaluation of 18 pregnant women with a singleton uncomplicated pregnancy, aged between 19 and 41 years ( $32 \pm 6$ year), who participated in the study after written informed consent. Each volunteer underwent an evaluation session every 4-6 weeks, starting from about the 22nd gestational week until delivery for a total of three to four acquisition sessions.

\section{Data collection}

Fetal echocardiography (including pulsed and Color Doppler) was performed (ESAOTE Biomedica AU5EPI) to exclude malformations, to assess fetal heart position, orientation, wall thickness, and to evaluate heart depth with respect to the maternal abdomen surface.

FMCG acquisitions were performed with a multi-channel system operating in magnetically shielded room. Each channel was a low-temperature dc-Super-conducting Quantum Interference Device (dc-SQUID) integrated magnetometer able to measure magnetic field variations ranging from 0.01 to $100 \mathrm{pT}$. Overall white noise density was about $5 f T / \sqrt{\mathrm{Hz}}$ above $60 \mathrm{~Hz}$ (19). The 55 sensing magnetometers covered a circular area of $230 \mathrm{~mm}$ diameter over the maternal abdomen.

Several acquisitions, lasting 5-10 $\mathrm{min}$ each, were performed during a fMCG session with $1 \mathrm{kHz}$ sampling frequency and recording bandwidth between 0.016 and $250 \mathrm{~Hz}$. Each acquisition produced a data set of $55 \mathrm{fMCG}$ recordings containing a mixture of maternal and fetal cardiac signals from which fetal cardiac traces could be retrieved. Women were asked to refer whether the fetus remained quiet or not during acquisition. Amagnetic electrodes were simultaneously used to monitor maternal ECG for data post-processing purposes.

\section{Data postprocessing}

ICA was used to reconstruct the fetal signals, because it permits a completely blind separation of signal sources starting from given mixed input signals, under the condition that the mixed traces derive from sources that are independent from each other $(20,21)$. This condition is verified in case of fMCG, and the Fixed Point ICA algorithm (FastICA), convenient from a computational point of view (22), was used because of its effectiveness in reconstructing reliable fetal traces with clear morphology from fMCG recordings $(17,18)$. Due to the ICA constraint that the number of mixed input signals must be greater than the number of independent sources, a maximum of eight raw fetal traces was reconstructed from each data set using a clustering procedure $(17,23)$. An adapted Kaiser filter was applied to smooth the traces, when necessary $(24,25)$.

\section{Cardiac time intervals analysis}

For each fMCG session, the reconstructed fetal traces showing the clearest waveform were used to estimate fCTI; at least 50 consecutive cardiac cycles were included in the analysis. The duration of $\mathrm{P}$ and $\mathrm{T}$ waves, QRS complex, PQ, PR, QT, QT corrected for the fetal heart rate $(\mathrm{QTc})$, and ST intervals was calculated following the standard definitions, as shown in Fig. 1 (26); ventricular frequency (RR) was also determined. The beginning and end of each wave were manually identified on all selected beats (Fig. 2); their values were automatically stored and used for statistical analysis. 


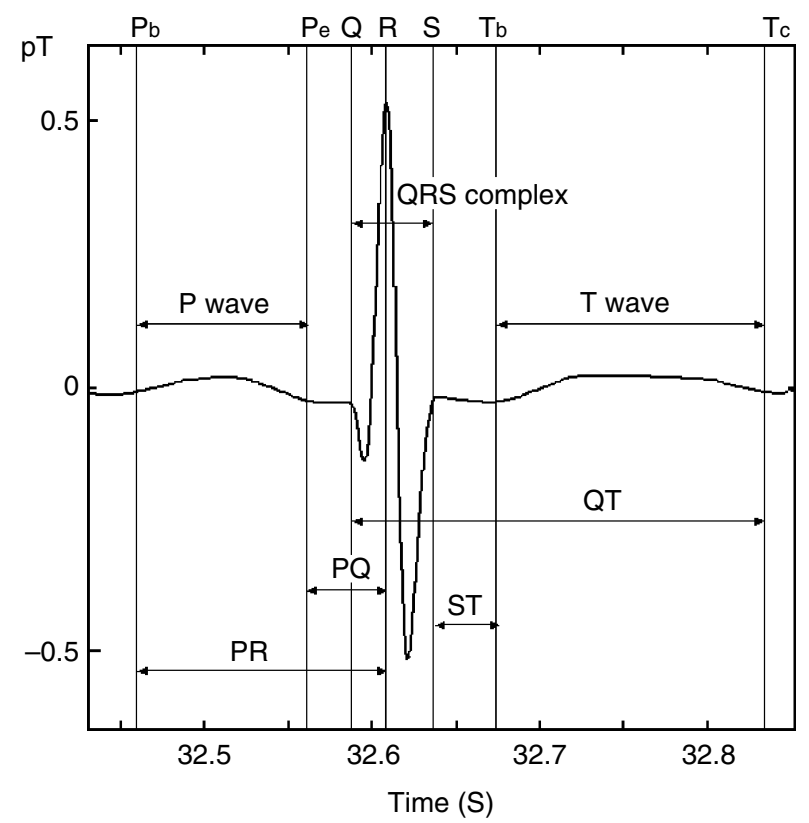

Fig. 1. QRS complex, $\mathrm{P}$ and $\mathrm{T}$ waves and fetal cardiac time intervals on a smoothed real-time fetal cardiac cycle of a trace reconstructed with Fixed Point ICA algorithm from fetal magnetocardiography recordings: definitions follow the recommended standards (26). Time is given in seconds from acquisition beginning and signal intensity is measured in $\mathrm{pT}$.

For each woman and for all acquisition sessions referring to her, intra-individual variability analysis of all fCTI was performed. Inter-individual variability analysis of fCTI was completed by grouping the results obtained on single patients in function of gestational age. The total period, ranging from the $22 \mathrm{nd}$ and the 37 th gestational week, was

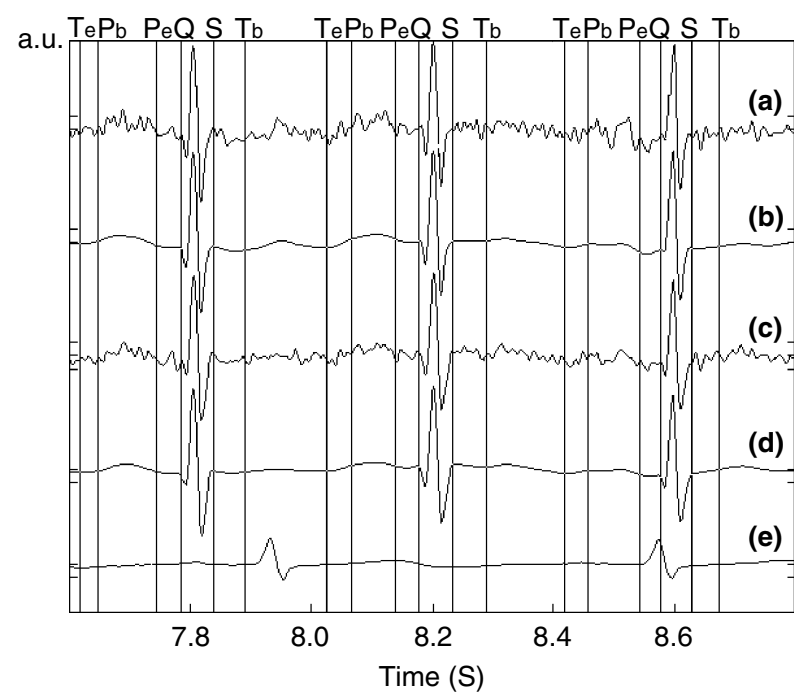

Fig. 2. Fetal cardiac traces reconstructed with Fixed Point ICA algorithm (a and c) and smoothed (b and d) using two clusters with opposite positions with respect to the fetal heart. Simultaneous maternal electrocardiogram (e) is provided to show the absence of maternal interference in fetal traces. Vertical lines on subsequent cardiac cycles identify onset and offset of $\mathrm{P}, \mathrm{QRS}$, and T waves and underline their simultaneity in all fetal signals. Time is given in seconds from acquisition beginning, and trace amplitude is in arbitrary units. divided in four subperiods of 4 weeks each. For each subperiod and for all intervals, we calculated the average and associated sampled standard deviation (SSD), which takes into account the total amount of fetal cycles used to obtain average values.

In order to compare our results with those obtained by other research groups, who estimated fCTI only on averaged beats, we calculated fCTI also on the averaged fetal beats obtained from the same rhythm strips used for beatto-beat analysis. For each time interval and for each gestational subperiod, we calculated an average percent variation $\overline{P D}$ between the results of the two methods as

$$
\overline{P D}=\frac{1}{N} \sum_{i=1}^{N}\left[\frac{\left(x_{a i}-\overline{x_{b i}}\right)}{x_{a i}}\right] 100
$$

where the ratio in the summation is: $\frac{\left(x_{a i}-\overline{x_{b i}}\right)}{x_{a i}}$

where $i$ identifies the data set, $N$ is the total number of data sets belonging to the analyzed subperiod, $x_{a i}$ is the interval duration estimated on averaged beats whereas $\overline{x_{b i}}$ is the average interval duration estimated on single cycles.

\section{Statistical analysis}

A linear regression model (one-tailed Pearson correlation) was used to quantify the relationship between fCTI and gestational age; 90, 95, and 98\% prediction intervals were calculated. Statistical significance was set at $P<0.05$. Individual trends of fCTI were drawn and compared with the prediction intervals to assess whether the latter ones could represent a large sample or not.

\section{Results}

Three women out of the 18 enrolled were excluded from the study because fetal arrhythmias occurred during fMCG. Therefore, 51 fMCG data sets of 55 recordings each were available for longitudinal analysis from 15 women; a total of more than 2600 fetal cardiac cycles were analyzed (Table I).

The overall detection rate of fetal signals reconstructed with FastICA was extremely good $(94 \%)$; its value for early gestation was remarkable $(80 \%)$ (Table I). Beat-to-beat estimate of time intervals on at least 50 consecutive beats was performed on all retrieved traces except two, which were recorded at 24 and 27 weeks and only permitted the calculation of RR variability due to their signal quality.

The longitudinal charts of normal fCTI during gestation, calculated for the entire population using the results of intra-individual analysis, are given in Table I. FCTI estimated on averaged fetal beats, and the related percent variations are shown in Table I to allow a comparison with the outcome of beat-to-beat analysis.

The results of simple linear regression analysis on fCTI obtained from beat-to-beat analysis and gestational age are summarized in Table II. An 
Table I. Longitudinal charts of normal fetal cardiac time intervals ( $\mathrm{fCTI}$ ) along pregnancy; they were calculated with intraindividual beat-to-beat analysis on more than 2600 cardiac cycles and on averaged fetal beats. Intervals duration and associated errors are given in milliseconds. QTc is expressed in ms ${ }^{1 / 2}$

\begin{tabular}{|c|c|c|c|c|}
\hline & \multicolumn{4}{|c|}{ Gestational subperiods (weeks) } \\
\hline & $22-25$ & $26-29$ & $30-33$ & $34-37$ \\
\hline Fetal magnetocardiography data sets & 10 & 15 & 13 & 13 \\
\hline Reconstructed fetal sets* & $8(80 \%)$ & $14(93 \%)$ & $13(100 \%)$ & $13(100 \%)$ \\
\hline
\end{tabular}

\begin{tabular}{|c|c|c|c|c|c|c|c|c|c|}
\hline & $\begin{array}{l}\text { Beat-to- } \\
\text { beat } \uparrow\end{array}$ & $\begin{array}{l}\text { Averaged } \\
\text { beatt }\end{array}$ & $\begin{array}{l}\text { Beat-to- } \\
\text { beat } \dagger\end{array}$ & $\begin{array}{l}\text { Averaged } \\
\text { beat: }\end{array}$ & $\begin{array}{l}\text { Beat-to- } \\
\text { beat } \dagger\end{array}$ & $\begin{array}{l}\text { Averaged } \\
\text { beat: }\end{array}$ & $\begin{array}{l}\text { Beat-to- } \\
\text { beat† }\end{array}$ & $\begin{array}{l}\text { Averaged } \\
\text { beat: }\end{array}$ & $\begin{array}{l}\text { Average percent } \\
\text { variation§ }\end{array}$ \\
\hline$P$ wave & $61(5)$ & $68(13)$ & $64(6)$ & $65(13)$ & $64(5)$ & $70(11)$ & 66 (4) & $71(8)$ & 12 \\
\hline$P Q$ & $32(4)$ & $38(6)$ & $33(6)$ & $38(10)$ & $34(5)$ & 41 (11) & $34(4)$ & 45 (13) & 26 \\
\hline PR & $93(4)$ & $106(13)$ & 97 (6) & 104 (17) & $98(8)$ & $111(11)$ & $100(9)$ & $115(21)$ & 16 \\
\hline QT & $263(8)$ & $230(24)$ & $270(11)$ & $208(29)$ & 285 (10) & $216(32)$ & $286(8)$ & $220(74)$ & -20 \\
\hline QRS & $54(4)$ & $54(6)$ & $53(3)$ & $56(7)$ & $53(3)$ & $57(9)$ & $60(2)$ & $48(7)$ & -2 \\
\hline ST & $68(6)$ & $69(26)$ & $73(7)$ & 46 (12) & $70(6)$ & $59(31)$ & $70(5)$ & 59 (28) & -13 \\
\hline T wave & $142(8)$ & 107 (13) & 151 (10) & $106(25)$ & $162(9)$ & $100(15)$ & $156(7)$ & $113(46)$ & -30 \\
\hline $\mathrm{RR}$ ब & $406(3)$ & & $432(4)$ & & $436(4)$ & & 437 (5) & & \\
\hline QTC & 413 (14) & & 409 (16) & & 432 (15) & & 434 (12) & & \\
\hline
\end{tabular}

*Number of sets of multiple fetal traces, retrieved with Fixed-Point ICA algorithm (FastICA), where P-QRS-T waves could be clearly identified on sequences of more than 50 beats; percentages of reconstructed data sets with respect to available fetal magnetocardiography (fMCG) data sets are given in parenthesis.

$\dagger$ Average values (SSD); figures obtained with beat-to-beat analysis.

†Average values (SD); figures refer to averaged beats calculated on the same rhythm strips used for beat-to-beat analysis.

$\S$ Ratio between the interval duration (average on the entire analyzed period) estimated on averaged beat and on sequences of more than 50 beats; positive and negative percent values indicate that intervals evaluated on averaged beats are longer and shorter, respectively.

ฯEstimate is possible only when single cardiac cycle are available.

example of the evolution of $\mathrm{P}, \mathrm{QRS}$, and T durations for a single fetus with respect to the general distributions is given in Fig. 3 to permit evaluating single trends relatively to normality ranges.

\section{Discussion}

FCTI throughout pregnancy can be quantified from isolated and repeated intra-individual fetal magnetocardiographic recordings. Intrinsic limitations of fMCG, such as significant dissimilarities in the morphology of fetal traces reconstructed from the same data set (27), the simultaneity of maternal and fetal QRS complexes that may infer erroneous fetal arrhythmias, residual maternal signal in reconstructed fetal traces, and peak-to-peak noise comparable to maximum fetal signal amplitude can reduce the overall quality of fetal rhythm strips; consequently, fCTI are generally estimated on averaged signals, and information on their variability is lost.

We have demonstrated in previous works (9, 17,18,25) that FastICA permits reconstructing reliable fetal traces with high detection rates (Table I) and unaltered morphology, so that fCTI could be assessed on single-cardiac cycles. For instance, QTc, which needs to be calculated on single beats and for this reason is rarely reported in the literature, was also quantified.

Table II. Simple linear regression model (one-tailed Pearson correlation) between cardiac time intervals and gestational age. Squared correlation coefficient $\left(r^{2}\right)$, slope and intercept of the regression line, statistical significance $(P)$, minimum and maximum interval values, given in milliseconds, are given

\begin{tabular}{|c|c|c|c|c|c|c|}
\hline & $r^{2}$ & Slope & Intercept & $P$ & $\operatorname{Min}(\mathrm{ms})$ & $\operatorname{Max}(\mathrm{ms})$ \\
\hline $\mathrm{RR}$ & 0.15 & 2.36 & 358.55 & $<0.05$ & 388 & 484 \\
\hline$P$ wave & 0.02 & 0.31 & 52.55 & NS & 49 & 78 \\
\hline$P Q$ & 0.00 & 0.05 & 31.51 & NS & 23 & 50 \\
\hline $\mathrm{PR}$ & 0.01 & 0.25 & 89.31 & NS & 74 & 124 \\
\hline QT & 0.10 & 2.41 & 204.35 & NS & 196 & 339 \\
\hline QTC & 0.06 & 2.64 & 342.82 & NS & 291 & 485 \\
\hline QRS & 0.13 & 0.47 & 41.27 & $<0.05$ & 41 & 64 \\
\hline ST & 0.00 & 0.06 & 68.78 & NS & 53 & 86 \\
\hline T wave & 0.11 & 1.45 & 105.88 & NS & 104 & 199 \\
\hline
\end{tabular}

NS, not significant. 

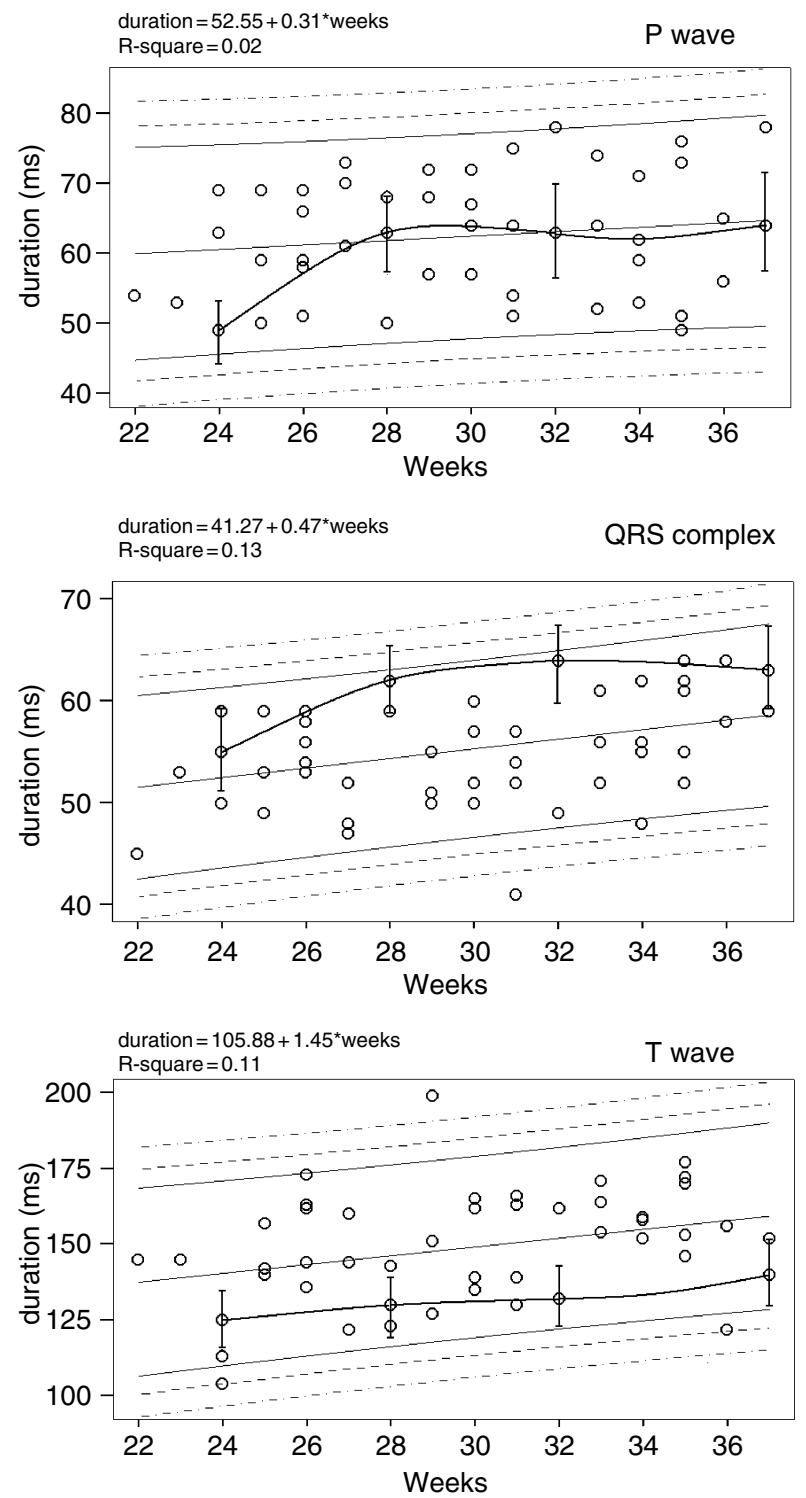

Fig. 3. Scatter plots of average duration of $\mathrm{P}$ wave (upper figure), QRS complex (middle figure), and $\mathrm{T}$ wave (lower figure) along gestation. Gestational age is given in weeks and interval duration in milliseconds. Regression line, regression equation, and prediction intervals of 90 (black line), 95 (dashed line), and 98\% (dash-dotted line) are given. As an example of individual trends, the evolution of $\mathrm{P}, \mathrm{QRS}$, and $\mathrm{T}$ waves within a single pregnancy is drawn on scatter plots; intra-individual variability is expressed by error bars.

The availability of clear and stable fetal signals allowed calculating fCTI on a huge number of consecutive beats with a precision increase (Table I) and the possibility to estimate, for each time interval, its intra-individual variability, which provides information amenable to be lost when fCTI are assessed on averaged traces. The longitudinal character of the study had two further positive effects: fCTI normality ranges could be quantified on a smaller group of women with respect to cross-sectional studies, and individual fCTI trends could be determined.
When comparing the outcome of our study with normality data developed cross-sectionally from a large number of women evaluated only once in their pregnancy $(11,13,14,16)$, a general agreement with the results by Grimm et al. (11) and Lowery et al. (13) was found, whereas some differences with those provided by Stinstra et al. (14) and Van Leeuwen et al. (16) were detected. These discrepancies, although within variability limits, regarded $\mathrm{P}$ wave, PQ, and QT (shorter duration in our estimates), the evolution of $\mathrm{P}$ wave and QRS complex along pregnancy, and the extent and evolution of $\mathrm{T}$ wave (longer duration in our estimates), although the authors reported a low-detection rate for $\mathrm{T}$ wave.

Remarkably, the differences between those findings and our results diminished when the comparison was performed with the fCTI estimates that we obtained using averaged beats. Indeed, the intervals related to $\mathrm{P}$ wave became longer and those related to $\mathrm{T}$ wave shorter, whereas QRS duration showed negligible differences. Since the same rhythm strips were used to estimate fCTI on single cycles and averaged beats, it is most likely that the observed differences in fCTI estimates should be ascribed to the averaging procedure. In fact, the physiological variability of fetal heart rate implies that the length of a cardiac wave may change in subsequent beats, inducing, in the averaged signal, an error that may increase with its distance from the alignment point ( $\mathrm{R}$ peak). This might explain the observed percent variations, particular large for the $\mathrm{T}$ wave, given its longer duration, weaker intensity, and frequent biphasic nature.

Residual differences may be due, as claimed by other authors $(14,28,29)$, to factors related to fetal behavioral state, position and orientation during fMCG, to the type and number of magnetic sensors used, and to environmental noise. Indeed, those conditions may induce important errors, of up to several milliseconds, in fCTI estimates. Conversely, fetal cardiac signals restored with FastICA showed to be independent on sensors configuration and unaffected by fetal movements, which were separated and disregarded as all other noise components, as shown in Fig. 2 (18).

The above considerations suggest that a beat-tobeat analysis of reliable fetal traces, although this procedure is lengthy and dependent on the investigator's expertise, should be preferred for a comprehensive estimate of fCTI. It is worth underlining that inter-individual charts of the fCTI evolution along gestation obtained from longitudinal intra-individual studies inherently contain more information than those attained with cross-sectional studies on averaged signals, although performed on larger 
populations. In fact, the proposed procedure permits evaluating a huge amount of cardiac cycles (over 2600 in the present study) and the small beat-tobeat cardiac activity variations, which are dumped during an averaging process, are quantified. When fCTI are estimated longitudinally within the same pregnancy, it is possible to define ranges and trends per patient, and, subsequently, reference trends in function of gestational age: in fact, the value of a time interval might be included in the normality ranges whilst its trend might be abnormal. The task of defining normality trends was not completely accomplished in our study due to the small number of intra-individual recordings available; however, given the good performances of FastICA, we are confident that larger series may be successfully collected and analyzed for a reliable assessment of fCTI duration, variability, and evolution along gestation.

\section{References}

1. Peters M, Crowe J, Piéri JF et al. Monitoring the fetal heart non-invasively: a review of methods. J Perinat Med 2001; 29: 408-16.

2. Lewis MJ. Review of electromagnetic source investigations of the fetal heart. Med Eng Phys 2003; 25: 801-10.

3. Taylor MJ, Smith MJ, Thomas $M$ et al. Non-invasive fetal electrocardiography in singleton and multiple pregnancies. Br J Obstet Gynaecol 2003; 110: 668-78.

4. Stinstra JG, Peters MJ. The influence of fetoabdominal tissues on fetal ECGs and MCGs. Arch Physiol Biochem 2002; 110: $165-76$.

5. Hosono T, Kanegawa T, Chiba Y, Kandori A, Tsukada K. The coincidence of fetal magnetocardiography and direct electrocardiography in a case of fetal atrial flutter due to intracardiac tumor. Fetal Diagn Ther 2002; 17: 331-3.

6. van Leeuwen P, Hailer B, Bader W, Geissler J, Trowitzsch E, Gronemeyer DH. Magnetocardiography in the diagnosis of fetal arrhythmia. Br J Obstet Gynaecol 1999; 106: 1200-8.

7. Menendez T, Achenbach S, Beinder $\mathrm{E}$ et al. Usefulness of magnetocardiography for the investigation of fetal arrhythmias. Am J Cardiol 2001; 88: 334-6.

8. Wakai RT, Strasburger JF, Li Z, Deal BJ, Gotteiner NL. Magnetocardiographic rhythm patterns at initiation and termination of fetal supraventricular tachycardia. Circulation 2003; 107: 307-12.

9. Comani S, Liberati M, Mantini D et al. Characterization of fetal arrhythmias by means of fetal magnetocardiography in three cases of difficult ultrasonographic imaging. Pacing Clin Electrophysiol 2004; 27: 1647-55.

10. Kähler C, Grimm B, Schleussner E et al. The application of fetal magnetocardiography (FMCG) to investigate fetal arrhythmias and congenital heart defects (CHD). Prenat Diagn 2001; 21: 176-82.

11. Grimm B, Kähler C, Schleussner E, Schneider U, Haueisen J, Seewald HJ. Influence of intrauterine growth restriction on cardiac time intervals evaluated by fetal magnetocardiography. Early Hum Dev 2003; 74: 1-11.

12. Horigome H, Shiono J, Shigemitsu S et al. Detection of cardiac hypertrophy in the fetus by approximation of the current dipole using magnetocardiography. Pediatric Res 2001; 50: 242-5.

13. Lowery CL, Campbell JQ, Wilson JD et al. Noninvasive antepartum recording of fetal S-T segment with a newly developed 151-channel magnetic sensor system. Am J Obstet Gynecol 2003; 188: 1491-7.

14. Stinstra J, Golbach E, van Leeuwen P et al. Multicentre study of fetal cardiac time intervals using magnetocardiography. Br J Obstr Gynecol 2002; 109: 1235-43.

15. Van Leeuwen P, Geue D, Lange S, Hatzmann W, Gronemeyer D. Changes in the frequency power spectrum of fetal heart rate in the course of pregnancy. Prenat Diagn 2003; 23: 909-16.

16. Van Leeuwen P, Lange S, Klein A, Geue D, Gronemeyer DH. Dependency of magnetocardiographically determined fetal cardiac time intervals on gestational age, gender and postnatal biometrics in healthy pregnancies. BMC Pregnancy Childbirth 2004; 4: 6.

17. Comani S, Mantini D, Pennesi P, Lagatta A, Cancellieri G. Independent component analysis: fetal signal reconstruction from magnetocardiographic recordings. Comput Methods Programs Biomed 2004; 75: 163-77.

18. Comani S, Mantini D, Lagatta A, Esposito F, Di Luzio S, Romani GL. Time course reconstruction of fetal cardiac signals from fMCG. Independent Component Analysis vs. Adaptive Maternal Beat Subtraction. Physiol Meas 2004; 25: 1305-21.

19. Della Penna S, Del Gratta C, Granata C et al. Biomagnetic systems for clinical use. Philosoph Magaz 2000; 80: 937-48.

20. Comon P. Independent component analysis - a new concept? Signal Processing 1994; 36: 287-314.

21. Bell AJ, Sejnowski TJ. Fast blind separation based on information theory. Proceedings of the International Symposium on Nonlinear Theory and Applications, Las Vegas, 1995, $43-7$.

22. Hyvärinen A. Fast and robust fixed-point algorithms for independent component analysis. IEEE Trans On Neural Networks 1999; 10: 626-34.

23. Mantini D, Comani S, Pennesi P et al. Fetal Tailoring of the Independent Component Analysis to multi-channel fMCG recordings for an optimal reconstruction of the fetal cardiac signal. Biomed Tech 2004; 48: 186-8.

24. Mitra SK, Kaiser F. Handbook for Digital Signal Processing. New York: John Wiley \& Sons 1993.

25. Comani S, Mantini D, Alleva G, Di Luzio S, Romani GL. Fetal Magnetocardiographic Mapping using Independent Component Analysis. Physiol Meas 2004; 25: 1459-72.

26. Grimm B, Haueisen J, Huotilainen $M$ et al. Recommended standards for fetal magnetocardiography. Pacing Clin Electrophysiol 2003; 26: 2121-6.

27. Burghoff B, Steinhoff U, Haberkorn W, Koch H. Comparability of measurement results obtained with multiSQUID-systems of different sensor configurations. IEEE Trans Appl Supercond 1997; 7: 3465-8.

28. Van Leeuwen P, Lange S, Klein A et al. Reproducibility and reliability of fetal cardiac time intervals using magnetocardiography. Physiol Meas 2004; 25: 539-52.

29. Hosono T, Chiba Y, Shinto M, Kandori A, Tsukada K. A fetal Wolff-Parkinson-White syndrome diagnosed prenatally by magnetocardiography. Fetal Diagn Ther 2001; 16: 215-7.

30. Kandori A, Hosono T, Chiba $\mathrm{Y}$ et al. Classifying cases of fetal Wolff-Parkinson-White syndrome by estimating the accessory pathway from fetal magnetocardiograms. Med Biol Eng Comput 2003; 41: 33-9.

31. Pirie AM, Wright J. Prenatal diagnosis of the Wolf-ParkinsonWhite syndrome by fetal magnetocardiography. $\mathrm{Br} \mathrm{J}$ Obstr Gynecol 2003; 110: 710 .

Address for correspondence:

Silvia Comani

Dipartimento di Scienze Cliniche e Bioimmagini,

Università 'G. D'Annunzio'

Via dei Vestini 33,

66013 Chieti, Italy

e-mail: comani@itab.unich.it 